\title{
Kaon Decays Reevaluated
}

\author{
A rigorous calculation of a matter-antimatter asymmetry in kaon decays \\ has twice the precision of a previous calculation, removing tension that \\ had existed between theory and experiment.
}

By Michael Schirber

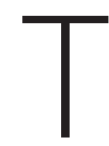

he kaon is unbalanced, as particles go. Decays of neutral kaons occur differently than those of its antiparticle partner, violating so-called charge-parity $(C P)$ symmetry. $C P$ violation in kaons is thought to be a good place to look for new physics beyond the standard model (see Synopsis: New Physics Possibilities from Kaon Decay). However, calculating the $C P$ violation expected from the standard model is difficult. The RBC and UKQCD particle physics collaborations have been working on this problem for two decades. Their latest joint computation [1], which has twice the statistical precision of their 2015 result, is in line with the experimental value, meaning more work will be needed to pinpoint possible new physics.

The first observation of $C P$ violation in kaons-made in 1964-was explained as an oscillation between two so-called $C P$-definite kaons, which behave in opposite ways when "flipped" by the CP symmetry. However, there exists another form of $C P$ violation, in which a $C P$-definite kaon decays directly into a set of particles with opposite $C P$. Experimentalists measured this direct $C P$ violation in the 1990s using decays of a

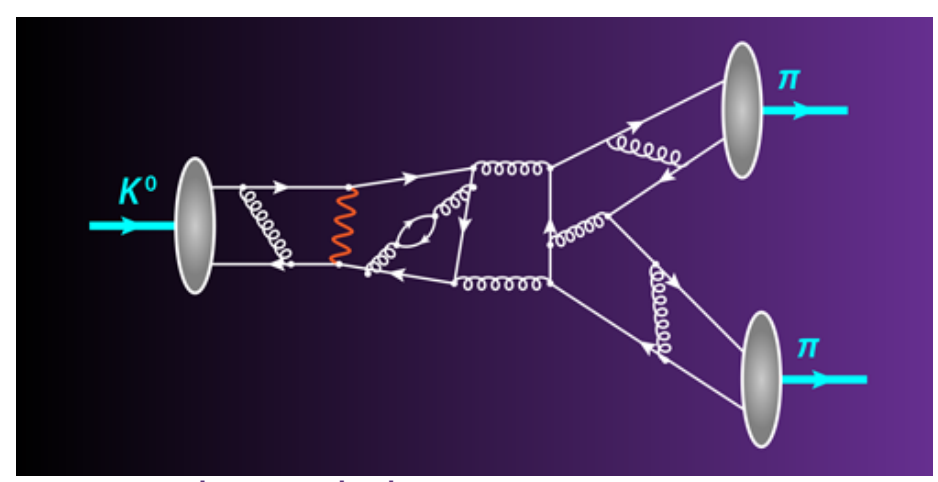

Credit: APS/Alan Stonebraker kaon into two pions. But subsequent theoretical work by the RBC and UKQCD teams suggested a possible disagreement between standard model predictions and experimental observations.

The RBC-UKQCD researchers have now improved those computations. The teams utilize the method of lattice quantum chromodynamics (QCD), which simulates strong interactions within small blocks of spacetime. The new simulations generated nearly 4 times more data than the previous effort. The team also corrected a systematic error from their earlier work, which neglected one of the ways that two-pion decays can be measured. The final result agrees with experiments, but beyond-standard-model physics might yet be revealed once the still-large theoretical error bars are further reduced.

Michael Schirber is a Corresponding Editor for Physics based in Lyon, France.

\section{REFERENCES}

1. R. Abbott et al. (RBC and UKQCD Collaborations), "Direct CP violation and the $\Delta I=1 / 2$ rule in $K \rightarrow \pi \pi$ decay from the standard model," Phys. Rev. D 102, 054509 (2020). 